\title{
Basic Quality Requirement and Basic Principle of Simultaneous Interpretation in English Translation
}

\author{
Tao Cui, Chundi Zhang \\ Foreign Language Department, Qiqihar Medical University, Qiqihar, 161006, China \\ Xexi123@163.com
}

Key words: English translation, Simultaneous interpretation, Basic quality, Basic principle

\begin{abstract}
Simultaneous interpretation as a translation mode with high efficiency and instantaneity is the most common interpretation way in international business conference in current society. Such translation form can not just effectively improve conference efficiency, but impose no impacts on speakers' thought and contribute to audiences to comprehend the contents of both parties. But such translation mode has very strict requirements for interpreters. This paper will analyze and discuss basic quality and working norms of English simultaneous interpreters in detail so as to promote development of Chinese simultaneous interpretation.
\end{abstract}

\section{Introduction}

Translation is a field involving a wide range, including written translation and oral interpretation as well as languages of various countries in the world. English written translation mainly refers to translation of newspapers, periodicals and magazines etc. Oral interpretation utilizes oral expression to transform language. But, in oral interpretation process, interpreters need to make sure there is no any delay according to requirements of working norms. According to different occasions, there are different kinds of professional terms and translation tasks. Simultaneous interpretation is the most difficult translation type with the strictest time limit and the highest requirements for language transformation speed and response speed in oral interpretation field. Hence, simultaneous interpretation workers need to own sufficient professional knowledge foundation and excellent flexibility in order to make sure they can adapt this job and offer superior interpretation services for important conferences.

\section{Meaning of simultaneous interpretation}

Simultaneous interpretation is the most difficult among all translation forms. Such translation form not just has strict requirements for interpreters' response and language transformation time, and also has high requirement for translation accuracy. Thus, simultaneous interpretation personnel should rapidly listen to, remember, digest, organize, transform and completely express the contents when both parties of a conference begin to speak. In other words, interpreters have to process all contents in their brain immediately and narrate the contents with the most accurate and proper language. So, one can imagine the complexity and difficulty. In the translation process, interpreters should not just digest speech contents, but also correct unofficial and non-normalized language in time. So, if interpreters do not own solid language foundation and flexibility, they cannot complete such work. Simultaneous interpretation is the highest stage of oral interpretation field and even the whole translation field, so the requirements for interpreters are naturally strict. But, simultaneous interpretation personnel also enjoy the highest treatment in translation field. Only such preferential treatment can stimulate interpreters to complete translation tasks in each conference with high quality. As China's economic development continuously tends to internationalization, domestic simultaneous interpretation development continuously advances. Especially in economic, business or diplomatic conferences, simultaneous interpretation personnel with solid knowledge base and relevant professional quality must be employed to convey conference contents by virtue of their flexibility and translation skills in order to make sure the conference is completed in a satisfying way and really 
promote China's economic development. If the qualification of simultaneous interpretation personnel is insufficient, conference content conveying may be not timely, and errors and even conference delay may be caused. Such situation will generate negative effects on the whole conference. If such negative effects happen in large conferences, national relations and economic development will be greatly affected negatively ${ }^{[1]}$.

As early as in late 1970s and early 1990s, some leading universities with iconic significance in Xiamen, Guangzhou and Beijing set up oral interpretation courses in English major and employed professors, scholars and excellent interpretation personnel with rich professional knowledge and oral English education experience for many years to teach simultaneous interpretation knowledge and skills for students. Under the historical background where economic development increasingly tends to internationalization, China's demand for foreign economic activities and international cultural exchange is also boosting. The contact with other countries in economy, culture and politics also becomes closer. Transnational business negotiation is naturally on the rise. Hence, the demand of domestic employment market for simultaneous interpretation personnel is in the rapid increasing stage, and the response is also very hot. Of course, such exuberant demand for talents brings both good opportunities and large challenges to Chinese simultaneous interpretation personnel. Various countries pay more and more attention to selection and cultivation of high-quality simultaneous interpretation talents. So, simultaneous interpretation talents can better adapt requirements of modern society for simultaneous interpretation talents, offer superior services for China's economic and trading activities and smooth development of diplomatic activities and promoting economic stability and sustainable development only through continuously enriching their knowledge and training their flexibility ability ${ }^{[2]}$.

\section{Basic requirements of simultaneous interpretation}

\section{Solid bilingual knowledge foundation and excellent flexibility}

Professional simultaneous interpretation personnel should own strong language expression talent and language sensitivity. Besides, they should own the ability to control native language and English. In addition to these, they also should own strong curiosity, exploration desire and strong attention to fresh things, because it is required to concentrate attention and ensure not to be affected so as to more accurately grasp the gist, organize and correct the language and guarantee expression accuracy in simultaneous interpretation process. High concentration is a key emphasis in work and also a difficult point in interpretation process. In view of instantaneity and flexibility features of simultaneous interpretation, the time for thinking and transformation is very limited. Thus, without solid language knowledge foundation and excellent flexibility, simultaneous interpretation personnel cannot transform and express language in time and even will delay conference course. Hence, only comprehensive talents with abundant language knowledge foundation and excellent flexibility who dabble in multi-aspect knowledge can be better qualified for this complex and demanding job ${ }^{[3]}$. As per simultaneous interpretation time requirement in international conference, except language foundation and flexibility, simultaneous interpretation personnel also should own very strong oral expression and linguistic organization ability. The speaking speed should be controlled within 250 words / minute, while general speaking speed is about 100 words / minute. Without professional training, one cannot be qualified for simultaneous interpretation work. Moreover, in speaking process, simultaneous interpretation personnel should ensure authenticity, logic and accuracy of the contents. The words should be expressed clearly and normatively. The intonation should be natural and fluent. The contents should be targeted, smooth and clean.

\section{Excellent psychological quality}

The contents of simultaneous interpretation are very extensive, involving economy, politics, culture, science and technology. Simultaneous interpretation is usually selected in large-scale conference. Thus, those predicating in interpretation will be nervous and have errors in speaking due to 
insufficient psychological quality. Even if they are quite familiar with the content, errors may also occur in the interpretation process due to tension ${ }^{[4]}$. So, good psychological basis of simultaneous interpreters is a major factor influencing interpretation with high efficiency, and strong emotion control ability is also quite necessary. Psychological state control is an effective way to better cope with all kinds of emergencies. Interpreters who can control their psychological states can keep calm in the face of any difficulties and complex situations. This has very great significance for understanding of conference contents and interpretation quality. Thus, simultaneous interpreters also should attach importance to improving their psychological quality while boosting their ability in order to make sure interpretation process proceeds.

\section{Teamwork concept}

Simultaneous interpretation team usually consists of 2-3 members. Each interpreter may interpret for about twenty minutes in each interpretation process, and they take turns to interpret. So, tacit cooperation and harmonious working environment of simultaneous interpretation team members are basic elements to make sure the whole interpretation process proceeds smoothly. Team awareness should be promoted as follows: firstly, the working time bucket and content should be arranged according to advantages of each interpreter so as to make sure advantages of each interpreter can be fully exerted; secondly, preparatory work should be listed as a key link. Detailed communication with interpretation team should be done in advance to give them sufficient preparation time and ensure smooth interpretation process and working efficiency; thirdly, in each interpretation exchange process, interpreters under break should pay attention to the work of team members and do assistant work for interpreters under work. Main contents of assistant work include avoiding external interference and recording conference contents etc.

\section{Working norms of simultaneous interpretation}

\section{Adjust anytime}

Self-adjustment ability is a basic ability that simultaneous interpretation personnel must own and also a core factor of maintaining work quality. To effectively adjust one's state, interpreters need to rationally adjust and organize new language information received, correct errors, amend the omission in order to complete high-quality interpretation. For example, in English, the sentences used to express position and time are usually placed at the end of a sentence as adverbial modifier, the positions of main clause and subordinate clause may be exchanged in the interpretation process. For instance, when translating "We will talk about this at last", if we translate it according to traditional sequence, the sentence is translated into “我们将要/讨论关于这个/在最后”. Such translation does not accord with Chinese sentence pattern. So, we need to adjust the sequence. Thus, this sentence should be translated into “这个问题我们将放在最后进行探讨”.

\section{Ahead of time properly}

Interpretation ahead of time properly mainly aims at the contents which can be obviously predicted in the interpretation process. Interpreters need to have obvious tendency to language information. When the sentence which can be predicted is not expressed completely, interpreters conduct interpretation ahead of time according to the content and specific context so as to let interpretation process is timelier. For example, when interpreting I accept your statement without reservation. (我完全接受您 的意见). According to specific expression requirements of such sentence pattern, interpreters may predict the content to be expressed. Thus, when hearing I accept your statement, interpreters may complete interpretation “我完全接受您的意见”[5]. 


\section{Development prospect of simultaneous interpretation}

At present, in the whole international market, follow-up development of simultaneous interpretation is optimistic and is regarded as a cause where there is lack of talents and is not short of money. Besides, simultaneous interpreters belong to rare human resources. It is most difficult to cultivate such kind of talents. According to relevant research results, the total number of world-wide professional simultaneous interpreters is about 2000. The demand for Chinese simultaneous interpretation talent resources exceeds supply. Hence, English has popularized at every corner in modern society, but the talents who can become simultaneous interpreters are very scarce. Simultaneous interpreters in minority languages are more rarely seen. As China communicates with various countries in the world frequently and China's international position is on the rise, international conferences that Chinese institutions and enterprises also become more and more. Each international conference cannot be held without simultaneous interpreters. Thus, simultaneous interpretation has good development prospect and competition in both domestic market and international market.

\section{Conclusion}

Simultaneous interpretation is a demanding, challenging and long-term occupation and also has many opportunities. Hence, Chinese English talents should enhance English knowledge foundation and professional ability, greatly improve English interpretation skills and enter simultaneous interpretation team in order to drive development of society and economy and achieve the value of English talents.

\section{Acknowledgments}

This paper is a key topic of teaching and research in Qiqihar Medical University: study on professional English course setting for medical students; No.: JY20141042

\section{References}

[1] Jiang Renji, On basic quality requirement and basic principle of simultaneous interpretation in English translation. Shenzhou (middle ten-day publication), 2014(8):97-97.

[2] Fang Kemin, Simultaneous interpretation skills and training. Journal of Beijing Polytechnic College, 2004,3(3):72-75.

[3] Yang Likun, Yang Lijun, On interpreting practice. Jingdezhen Comprehensive College Journal, 2005,20(1):51-52.

[4] Guo Yuqi, On some methods and skills of simultaneous interpretation. Teaching Russian In China, 2010,29(1):67-71.

[5] Tang Aiyan, Communicative strategy in simultaneous interpretation. Chinese Science \& Technology Translators Journal, 2009,22(3):19-23. 